\title{
REVIEW \\ Pre-Exposure Prophylaxis: A Narrative Review of Provider Behavior and Interventions to Increase PrEP Implementation in Primary Care
}

\author{
Andrew Silapaswan, $\mathrm{BS}^{1,2,3}$, Douglas Krakower, $\mathrm{MD}^{1,4,5}$, and Kenneth H. Mayer, $\mathrm{MD}^{1,3,4,5}$ \\ ${ }^{1}$ Fenway Health, The Fenway Institute, Boston, MA, USA; ${ }^{2}$ New York Medical College, Valhalla, NY, USA; ${ }^{3}$ Harvard T.H. Chan School of Public Health, \\ Boston, MA, USA; ${ }^{4}$ Beth Israel Deaconess Medical Center, Boston, MA, USA; ${ }^{5}$ Harvard Medical School, Boston, MA, USA.
}

Since FDA approval of HIV pre-exposure prophylaxis (PrEP) for HIV prevention, attention has been focused on PrEP implementation. The CDC estimates that 1.2 million U.S. adults might benefit from PrEP, but only a minority are using PrEP, so there is a significant unmet need to increase access for those at risk for HIV. Given the large numbers of individuals who have indications for PrEP, there are not enough practicing specialists to meet the growing need for providers trained in providing PrEP. Moreover, since PrEP is a preventive intervention for otherwise healthy individuals, primary care providers (PCPs) should be primary prescribers of PrEP. There are important clinical considerations that providers should take into account when planning to prescribe PrEP, which are highlighted in the clinical case discussed. A growing body of research also suggests that some providers may be cautious about prescribing PrEP because of concerns regarding its "real-world" effectiveness, anticipated unintended consequences associated with its use, and ambiguity as to who should prescribe it. This review summarizes findings from studies that have assessed prescriber behavior regarding provision of PrEP, and offers recommendations on how to optimize PrEP implementation in primary care settings. Development and dissemination of educational interventions for PCPs and potential PrEP users are needed, including improved methods to assist clinicians in identifying appropriate PrEP candidates, and programs to promote medication adherence and access to social and behavioral health services. PCPs are wellpositioned to prescribe PrEP and coordinate healthrelated services to improve the sexual health of their patients, but tailored educational programs are needed.

KEY WORDS: pre-exposure prophylaxis (PrEP); HIV/AIDS; prevention; men who have sex with men (MSM); primary care.

$\mathrm{J}$ Gen Intern Med 32(2):192-8

DOI: $10.1007 / \mathrm{s} 11606-016-3899-4$

(c) Society of General Internal Medicine 2016

A lthough U.S. HIV incidence has remained stable in recent years, with approximately 50,000 new infections annually, specific populations remain disproportionately

Received June 8, 2016

Revised September 23, 2016

Accepted September 28, 2016

Published online October 19, 2016 affected. ${ }^{1}$ Men who have sex with men (MSM) bear a disproportionate burden of HIV, and in 2014 accounted for $67 \%$ of new diagnoses. ${ }^{2}$ Lifetime HIV incidence rates for African American and Hispanic/Latino MSM are 50 and $25 \%$, respectively. ${ }^{2}$ Increasing rates of HIV incidence in MSM underscore the need for improved access to effective HIV prevention options. In 2012, the U.S. Food and Drug Administration (FDA) approved the once-daily fixed-dose combination of tenofovir disoproxil fumarate and emtricitabine (TDF-FTC) for use as oral pre-exposure prophylaxis (PrEP) for HIV prevention. ${ }^{3}$ Since FDA approval and publication of the Centers for Disease Control and Prevention (CDC) clinical practice guidelines in 2014, attention has been focused on PrEP implementation, particularly by primary care providers (PCPs).

Multiple studies have assessed provider attitudes, intentions, and behaviors regarding PrEP, and have identified potential barriers to PrEP implementation. Data on providerreported concerns have largely been gathered from HIV specialists, ${ }^{4}$ infectious disease physicians, ${ }^{5,6}$ and other providers in large urban centers. ${ }^{7-11}$ Current understanding of prescriber behavior may be limited by the geographic and specialized nature of those interviewed.

The following case describes the clinical history of a patient engaged in primary care in Boston, Massachusetts, and highlights several key clinical considerations when prescribing PrEP. The subsequent review of provider-reported barriers includes recommendations on strategies for increasing the rollout of PrEP in primary care. Given the disproportionate burden of new HIV infections among MSM, this review will focus primarily on the evidence pertaining to MSM and the sexual risk of HIV acquisition.

\section{CLINICAL EXAMPLE ILLUSTRATING CONSIDERATIONS WHEN PRESCRIBING PREP}

Mr. A is a 49-year-old man who presented for routine care with his PCP. He had been in a long-standing sexual relationship with a woman who was HIV-infected, and expressed concerns about his risk for HIV. He engaged in condomless oral and penile-vaginal sex with his female partner twice a month. His partner did not use antiretroviral medication. He also reported sexual contact with men of unknown HIV status 
about twice per year, including condomless insertive and receptive oral and anal sex. Although he reported sexual activity with men, he identified as heterosexual. Mr. A had been treated twice for sexually transmitted infections (STI) and was prescribed HIV post-exposure prophylaxis twice after condomless sex with his female partner. After a discussion about PrEP with his PCP, he began PrEP after being screened for bacterial STI and found to be HIV-uninfected, with normal renal function and a negative hepatitis B surface antigen test finding.

During his first year of PrEP use, Mr. A remained HIVuninfected and did not acquire any other STIs. After about 12 months of PrEP use, however, he discontinued PrEP after experiencing housing instability and depression. Mr. A's history illustrates several challenges that providers should consider when prescribing PrEP, as they may affect long-term adherence. His sexual history also demonstrates how sexual identity may not be consistent with sexual behavior. Although PCPs are well-positioned to prescribe PrEP, because many atrisk persons present for primary care (as opposed to specialty clinics), studies have found that some providers remain cautious about prescribing PrEP. The following review of provider-reported barriers discusses concerns regarding the "real-world" efficacy of PrEP, potential concerns regarding its implementation, and uncertainty as to who should be the primary prescribers of PrEP. ${ }^{5,9,12,13}$ Finally, recommendations on how to optimize PrEP implementation within primary care are discussed.

\section{BARRIERS TO PREP "REAL-WORLD” EFFECTIVENESS}

\section{PrEP Efficacy and Medication Adherence}

Efficacy studies have demonstrated that PrEP has the potential to prevent many HIV infections, and medication adherence is critical to achieving maximal protection. ${ }^{14-22}$ The iPrEx study, a placebo-controlled randomized study of $2500 \mathrm{MSM}$ and transgender women, demonstrated a $44 \%$ reduction in HIV incidence in those assigned to TDF-FTC, and an estimated $99 \%$ reduction in those whose drug levels suggested daily adherence. ${ }^{14,23}$ Efficacy estimates in other placebo-controlled studies of daily TDF-FTC PrEP vary greatly, with medication adherence being the major predictor of efficacy. ${ }^{14-22}$ Some providers have cited suboptimal efficacy in several trials as a reason not to consider PrEP as a mainstay for prevention. ${ }^{4-11}$ However, participants in early studies were told they might receive a placebo, and PrEP efficacy was not proven. In subsequent studies, when individuals were educated about the importance of adherence, efficacy results were higher. A randomized controlled trial of MSM accessing services in British STI clinics found that those who had access to PrEP had $86 \%$ decreased HIV incidence compared to those assigned to a wait list. ${ }^{24} \mathrm{~A}$ clinic-based study of PrEP use by over 650 MSM in San Francisco found high adherence rates and no new HIV infections. ${ }^{25,26}$ However, PrEP adherence has remained a challenge for some individuals. ${ }^{27}$ A study of young MSM aged 18 to 22 years found that adherence attenuated over time, underscoring the need for ongoing medication adherence support. ${ }^{28}$ Women may need more consistent daily adherence than MSM because of the slower pharmacokinetics of tenofovir accumulation in cervicovaginal tissue compared to rectal mucosa. ${ }^{29}$

In the IPERGAY study, 414 MSM and transgender women were randomized to receive pericoital TDF-FTC as PrEP or placebo. ${ }^{15}$ Participants randomized to receive TDF-FTC were directed to take two pills of TDF-FTC 2 to $24 \mathrm{~h}$ prior to sexual activity, followed by a third pill $24 \mathrm{~h}$ after the first dose, and a fourth pill $24 \mathrm{~h}$ later. Study results demonstrated an $86 \%$ reduction in HIV incidence. ${ }^{15}$ Participants used an average of 15 pills per month, ${ }^{15}$ so it is not known whether pericoital PrEP would confer HIV risk reduction benefits to those who have less frequent HIV exposure. Despite ongoing research on pericoital PrEP and other alternative dosing regimens, only daily TDF-FTC is currently FDA-approved for PrEP.

PrEP users may benefit from routine adherence counseling and support services. CDC clinical guidelines recommend that prescribers perform regular medication adherence counseling and develop strategies with patients to optimize adherence. ${ }^{30}$ Adopting a framework of neutral adherence assessment is recommended to help providers obtain more accurate patient information regarding medication adherence. ${ }^{31}$ Measuring blood, hair, or urine levels of tenofovir to monitor adherence is under study, and could potentially offer methods for monitoring adherence in clinical practice. ${ }^{32}$

\section{Logistical Concerns}

Providers have also reported logistical concerns when prescribing PrEP, ${ }^{9}$ particularly time constraints, which can make it challenging for PCPs to complete risk behavior assessment and medication adherence counseling during standard brief clinical encounters. Risk behavior assessments are critical, however, to identify appropriate candidates for PrEP. Innovative approaches to assessing risk, including computer-based risk assessments that patients can complete independently and prior to clinician visits, are being explored.

Insurance coverage and out-of-pocket costs of PrEP have also been cited as barriers to implementation. ${ }^{9}$ The average wholesale price of TDF-FTC for PrEP is approximately $\$ 1425$ per month. ${ }^{33}$ While most private insurance companies and state Medicaid programs provide coverage for PrEP, ${ }^{13,33}$ copayments vary among individual insurance plans, so the PrEP package expense can be daunting for under-insured individuals.

Gilead Sciences, the manufacturer of TDF-FTC, has developed the "Truvada for PrEP Medication Assistance Program" to subsidize costs for uninsured individuals. ${ }^{34}$ However, many patients have annual incomes exceeding income eligibility criteria, thereby limiting access. ${ }^{13}$ Ideally, clinicians or case workers can review cost-sharing 
requirements with patients prior to initiating PrEP and can refer patients to medication assistance programs as needed. Determining cost-sharing requirements based on individual health plans may be a logistical challenge for PrEP providers. To help identify these barriers, providers may benefit from partnering with social service organizations to determine cost-sharing and insurance coverage requirements for specific plans.

\section{POTENTIAL UNINTENDED CLINICAL CONSEQUENCES}

Providers may cite other concerns as reasons they are cautious about prescribing PrEP, including selection for antiretroviral drug resistance, medication toxicities, and behavioral disinhibition and STIs. ${ }^{4-11}$

\section{Antiretroviral Drug Resistance}

Data from PrEP clinical trials indicate that development of antiretroviral resistance was rare for those who acquired HIV while taking PrEP. ${ }^{35,36}$ In the original efficacy trials, few patients developed drug resistance after acquiring HIV, and transmitted drug resistance was rare; the few cases of resistance tended to occur among patients who initiated PrEP with undiagnosed acute HIV infection (antibody negative when enrolled, but subsequently determined to be viremic). ${ }^{14-21}$ Although these data and modeling studies ${ }^{37,38}$ suggest that PrEP may have a minimal contribution to antiretroviral resistance at the population level, these observations are based on limited data, so additional studies assessing PrEP-associated drug resistance are needed. ${ }^{39}$ One recent report of transmitted multiclass drug-resistant HIV occurred in a patient who was adherent to PrEP. ${ }^{40}$ This case report indicates that PrEP failure because of resistance can happen, but is rare when the aggregate PrEP experience is considered. Patients should be informed that TDF-FTC PrEP is highly effective when used consistently, but not $100 \%$ effective. Since antiretroviral resistance could occur in individuals who experience seroconversion and continue to take PrEP, baseline and routine HIV testing every 3 months (and sooner if acute infection is suspected) are indicated to avoid selection for resistant HIV. Patients who discontinue and later decide to restart PrEP should undergo the same baseline evaluation prior to resumption of PrEP. ${ }^{30}$

\section{PrEP Drug Toxicity}

A minority of PrEP users have reported symptoms when using TDF-FTC, but were more likely to report headache, nausea, and diarrhea than those using placebos. This is often referred to as "startup syndrome," and symptoms usually resolve in the first few days to weeks after PrEP initiation. ${ }^{41}$ Despite the favorable safety profile, some prescribers have cited concerns about PrEP-related toxicities, ${ }^{4-11}$ as studies have demonstrated a weak association between TDF and decreased renal function ${ }^{42-44}$ or modest, reversible loss of bone mineral density (BMD). ${ }^{45-47}$ Although long-term follow-up data are needed, efficacy and safety studies of TDF with and without FTC for PrEP did not demonstrate significant differences in rates of clinically significant renal events between treatment and control groups. ${ }^{14-21}$ Elevated creatinine levels usually returned to baseline after discontinuation of TDFFTC. ${ }^{14-21}$ Studies of TDF with and without FTC for use as PrEP found a $1 \%$ decrease in BMD in a minority of patients that tended to occur during the first several months after initiation. ${ }^{14,17,48}$ Progressive loss of BMD did not occur, and usually returned to baseline several months after discontinuing PrEP. ${ }^{49}$ There was also no increase in fracture rates over the 1-2 year-follow-up period in treatment arms compared to control. ${ }^{48}$ Clinical follow-up in the first few weeks after initiating PrEP is recommended to assess for side effects, and regular testing for renal function every 3 to 6 months, particularly for those at risk of renal injury. Current guidelines recommend that patients with a history of bone fractures or who have risk factors for osteoporosis should be referred for appropriate consultation and management prior to initiating PrEP. ${ }^{30}$ Additional PrEP formulations with improved side effect profiles, such as tenofovir alafenamide fumarate (TAF), are currently being evaluated for HIV treatment. ${ }^{50}$ However, pharmacokinetic studies have found that TAF achieves lower tissue concentrations in anogenital mucosa than does TDF, which might impact its effectiveness for use as PrEP. ${ }^{51} \mathrm{~A}$ head-to-head comparison of TAF-FTC and TDF-FTC is being initiated to determine whether TAF-FTC should be used as PrEP.

\section{Behavioral Disinhibition and Sexually Transmifted Infections}

Behavioral disinhibition or risk compensation, whereby persons using PrEP might increase their sexual risk behaviors, is a potential unintended consequence of PrEP that could increase the risk of STI transmission. Compensatory increases in sexual risk behavior were not observed in PrEP efficacy trials. ${ }^{14-21}$ The early studies involved intensive risk reduction counseling, however, and participants may have underreported sexual risk behaviors due to social desirability bias. ${ }^{14,15,52,53} \mathrm{~A}$ recent observational study of MSM in primary care noted that some MSM who used PrEP reported an increase in the number of sexual partners and a decrease in condom use, though behavioral changes could not be definitively attributed to PrEP use, given the lack of a control group. ${ }^{25}$ Long-term data on behavioral practices associated with PrEP outside of research settings are needed. It appears that most PrEP users had not consistently used condoms prior to starting PrEP, and did not start doing so thereafter. This pattern of behavior suggests "risk maintenance" and not risk compensation. Nonetheless, as PrEP does not prevent transmission of other STIs, PrEP 
must be delivered in combination with other prevention methods (e.g. condoms) and regular STI screening to prevent and/or promptly treat incident STIs, given that high rates of incident bacterial STI have been seen recently in PrEP cohorts. ${ }^{25,54,55}$

\section{PURVIEW PARADOX}

Although CDC clinical guidelines have increased knowledge and awareness of PrEP, contradictory beliefs exist regarding appropriate PrEP providers, referred to as a "purview paradox." Some providers have indicated that PrEP may not fall within their clinical domain. PCPs have described unfamiliarity with prescribing antiretrovirals and prescribing PrEP, ${ }^{9}$ whereas some HIV specialists have indicated that they primarily provide care to HIV-infected patients and have had few high-risk HIV-uninfected patients.

Uptake of PrEP was slow during the early phase of PrEP implementation $;{ }^{56}$ however, recent data indicate more widespread use. ${ }^{57,58}$ Despite an increase in PrEP utilization, the CDC estimates that 1.2 million adults can benefit from PrEP, of which 492,000 are adult MSM. ${ }^{59}$ As there are only 6200 infectious disease physicians in the U.S. involved in patient care ${ }^{60}$ specialists alone could not provide PrEP to these large numbers of patients. Thus, engagement of PCPs will be critical in scaling up access to PrEP.

Although most providers are aware of PrEP, knowledge and awareness differ by specialty. Provider knowledge of PrEP and CDC clinical guidelines appears to be higher among HIV specialists than among generalists. ${ }^{8-10,61,62}$ The fact that generalists are less aware of PrEP underscores the need for improved training, since the majority of at-risk patients are likely to seek health care from PCPs and not HIV specialists. ${ }^{9}$ PCPs are well-positioned to identify individuals who may be appropriate candidates for PrEP. Dissemination of PCP educational interventions to increase knowledge of PrEP is needed to close the information gap between generalists and HIV specialists.

\section{RECOMMENDATIONS TO INCREASE PREP IMPLEMENTATION IN MSM}

To improve the rollout of PrEP, multilevel interventions are needed to address provider-reported barriers to PrEP implementation. These include provider- and patient-level interventions. A comprehensive implementation strategy should include provider training along with community engagement to support medication adherence in high-risk MSM.

\section{Provider Educational Interventions}

Studies demonstrate that knowledge of PrEP efficacy correlates with willingness to provide PrEP. ${ }^{9}$ Furthermore, increased knowledge of PrEP is associated with having previously prescribed PrEP and future intent to prescribe PrEP. ${ }^{61}$
Evidence-based educational interventions for providers are needed that include information on comprehensive sexual healthcare, behavioral risk assessments, PrEP safety and efficacy data, and clinical practice guidelines. Such interventions could address providers' concerns that may be limiting their engagement in PrEP implementation.

Data also indicate that educational interventions have the potential to increase the number of providers prescribing PrEP. After individual-level presentations on PrEP to infectious diseases providers and PCPs in New York City, $13 \%$ of providers subsequently prescribed PrEP for the first time at a 5-8-week follow-up visit. ${ }^{63}$ However, infectious disease physicians were more willing than PCPs to prescribe PrEP after receiving education, so educational interventions may need to be tailored to generalists. ${ }^{63}$ It may be helpful to link potential prescribers to local organizations that provide consultation and training about sexual and gender minority health. One online resource is the National Center for LGBT Health Education (www.lgbthealtheducation.org). Establishing these partnerships could provide clinicians with the support needed to identify and address clinical and social barriers to prescribing PrEP.

\section{Optimizing Individual Knowledge and Medication Adherence Through Community Engagement}

In addition to provider interventions, patient-oriented programs are needed to optimize PrEP implementation. Educational interventions containing PrEP-related information should also be directed to MSM and other populations at higher risk of HIV acquisition. This can facilitate selfdirected referrals for initiating PrEP. PrEP users may also require adherence support services offered by communitybased organizations (CBOs). PCPs should be aware of local resources supporting PrEP adherence.

Ongoing research to improve PrEP adherence is underway. Researchers at Fenway Health, a community health center in Boston, have developed a PrEP support tool based on text messaging and adherence counseling to promote medication adherence. Study results have demonstrated that $84 \%$ of participants who received a PrEP adherence intervention, which consisted of four weekly counseling sessions delivered by a nurse, had drug levels consistent with daily PrEP use 6 months post-intervention. ${ }^{64}$ Provider counseling in combination with medication adherence interventions may have the greatest impact in maximizing PrEP adherence, so it will be important for PCPs to be informed about local resources and $\mathrm{CBO}$ programs that provide ongoing support.

\section{Improved Identification of Appropriate Candidates for PrEP}

The current unmet demand for PrEP also requires improved methods of identifying appropriate candidates for PrEP within primary care. However, providers may be 
uncomfortable discussing sexual behavior and HIV risk with patients, ${ }^{65}$ which may limit access to PrEP. Data from one HIV clinic indicated that $46 \%$ of respondents felt uncomfortable discussing their sexual practices with their PCP. ${ }^{66}$ Furthermore, PCPs may not routinely ask questions about sexual orientation and gender identity ( $\mathrm{SO} / \mathrm{GI})$, or conduct routine sexual risk behavior assessments. ${ }^{67}$ As a result, patients who would be eligible candidates for PrEP may not be identified.

Novel approaches to streamlining behavioral risk assessments and incentivizing providers to routinely ask $\mathrm{SO} / \mathrm{GI}$ questions could lead to improved identification of PrEP candidates. Risk screening algorithms that use patient-reported behaviors to generate estimates of HIV risk have been developed to identify patient candidates for PrEP. ${ }^{68,69}$ It may also be beneficial for providers to ask patients $\mathrm{SO} / \mathrm{GI}$ questions as part of comprehensive care. Proposed changes to the Health Resources and Services Administration 2016 Uniform Data System reporting include routine collection of SO/GI data. ${ }^{70}$ The Centers for Medicare and Medicaid Services will also require electronic health records certified under the meaningful use incentive program to include $\mathrm{SO} / \mathrm{GI}$ data fields beginning in $2017 .^{71}$ By increasing routine $\mathrm{SO} / \mathrm{GI}$ data collection, providers should be better able to identify appropriate candidates for PrEP.

\section{Coordination of Care and Other Health- Related Services in Primary Care}

As the U.S. health care system continues to undergo reform, it would be desirable to incentivize PCPs to conduct routine HIV risk assessments and to oversee the provision of PrEP as part of value-based care. This could potentially occur in patient-centered medical homes that are designed to optimize coordinated care and preventive health. Provision of PrEP requires behavioral risk assessment, medication adherence counseling, and other health-related services. These include HIV testing at least every 3 months, other STI testing at least every 6 months, routine laboratory testing, and coordination of support services. ${ }^{30}$ Although some PCPs report challenges in the provision and coordination of PrEP-related services, ${ }^{4-11}$ primary care clinics have the infrastructure to provide coordinated longitudinal care that is needed for PrEP. Evaluations of PrEP implementation in multiple clinical settings offer evidence that non-HIV specialists can successfully deliver PrEP in primary care settings, and future efforts can build upon these early successes. $^{72}$

PCPs also have the opportunity to discuss PrEP in the context of overall health. These conversations allow providers and patients to discuss why patients may engage in high-risk behaviors and to develop strategies to reduce HIV risk behavior. Bidirectional communication can allow for identification of root causes of HIV-related risk behaviors such as depression, ${ }^{73,74}$ substance use, ${ }^{75-77}$ stigma, and discrimination. ${ }^{78,79}$ Lastly, PCPs are well trained in shared decision-making and providing holistic care to patients, which are ideal attributes for delivering a bio-behavioral intervention such as PrEP.

\section{CONCLUSION}

Despite efforts to increase access to PrEP, there remains a large unmet demand. Expanding access within primary care will be critical for scaling up PrEP implementation. Additional research on implementation strategy is needed to increase access to PrEP and other HIV prevention services. Interventions to enhance PrEP implementation should focus on dissemination of provider educational interventions, improved identification of appropriate candidates for PrEP, and community engagement regarding medication adherence and access to support services. PCPs are well-positioned to prescribe PrEP and to coordinate health-related services in the context of longitudinal care that addresses overall sexual health. Moving forward, coordinated efforts to increase the rollout of PrEP within primary care may have the greatest effect on increasing access to PrEP for MSM and other appropriate candidates for PrEP.

Corresponding Author: Kenneth H. Mayer, MD; Fenway HealthThe Fenway Institute, 1340 Boylston St., Boston, MA 02215, USA (e-mail: KMayer@fenwayhealth.org).

\section{Compliance with Ethical Standards:}

Conflict of Interest: Dr. Mayer and Dr. Krakower have received unrestricted research grants from Gilead Sciences, and Dr. Mayer has received an unrestricted research grant from ViiV Health Care. Mr. Silapaswan reports no conflicts of interest.

\section{REFERENCES}

1. Prejean J, Song R, Hernandez A, et al. Estimated HIV incidence in the United States, 2006-2009. PLoS ONE. 2011;6(8):e17502.

2. Centers for Disease Control and Prevention. HIV among gay and bisexual men. Available at: http://www.cdc.gov/hiv/group/msm/. Accessed September 29, 2016.

3. U.S. Food and Drug Administration. FDA approves first drug for reducing the risk of sexually acquired HIV infection [Press Release]. Available at: http://www.fda.gov/NewsEvents/Newsroom/PressAnnouncements / ucm312210.htm. Accessed September 29, 2016.

4. Tellalian D, Maznavi K, Bredeek UF, Hardy WD. Pre-exposure prophylaxis (PrEP) for HIV infection: results of a survey of HIV healthcare providers evaluating their knowledge, attitudes, and prescribing practices. AIDS Patient Care STDs. 2013;27(10):1-7.

5. Karris MY, Beekmann SE, Mehta SR, Anderson CM, Polgreen PM. Are we prepped for preexposure prophylaxis (PrEP)? Provider opinions on the real-world use of PrEP in the United States and Canada. Clin Infect Dis. 2014;58(5):704-12.

6. Krakower DS, Beekmann SE, Polgreen PM, Mayer KH. Frontline practices with HIV prevention: A survey of U.S. infectious disease physicians. Conference on retroviruses and opportunistic infections [abstract 1083]. Seattle, Washington: 2015; 23-26 February.

7. Arnold EA, Hazelton P, Lane T, et al. A qualitative study of provider thoughts on implementing pre-exposure prophylaxis (PrEP) in clinical settings to prevent HIV infection. PLoS ONE. 2012;7(7):e40603.

8. White JM, Mimiaga MJ, Krakower DS, Mayer KH. Evolution of Massachusetts physician attitudes, knowledge, and experience regarding 
the use of antiretrovirals for HIV prevention. AIDS Patient Care STDs. 2012;26(7):395-405.

9. Krakower DS, Ware N, Mitty JA, Maloney K, Mayer KH. HIV providers' perceived barriers and facilitators to implementing preexposure prophylaxis in care settings: a qualitative sutdy. AIDS Behav. 2014;18(9):1712-21.

10. Krakower DS, Oldenburg CE, Mitty JA, et al. Knowledge, beliefs and practices regarding antiretroviral medications for HIV prevention: results from a survey of healthcare providers in New England. PLoS ONE. 2015;10(7):1-15.

11. Hoffman S, Guidry JA, Collier KL, et al. A clinical home for preexposure prophylaxis: diverse health care providers' perspectives on the "purview paradox". J Int Assoc Provid AIDS Care. 2016;15(1):59-65.

12. Krakower DS, Mayer KH. The role of healthcare providers in the roll out of preexposure prophylaxis. Curr Opin HIV AIDS. 2016;11(1):41-8.

13. Mayer KH, Hosek S, Cohen $\mathbf{S}$, et al. Antiretroviral pre-exposure prophylaxis implementation in the United States: a work in progress. J Int AIDS Soc. 2015;18(4 Suppl 3):1-7.

14. Grant RM, Lama JR, Anderson PL, et al. Preexposure chemoprophylaxis for HIV prevention in men who have sex with men. N Engl J Med. 2010;363(27):2587-99.

15. Molina JM, Capitant C, Spire B, et al. On-demand preexposure prophylaxis in men at high risk for HIV-1 infection. N Engl $\mathrm{J}$ Med. 2015;373(23):2237-46.

16. Thigpen MC, Kebaabetswe PM, Paxton LA, et al. Antiretroviral preexposure prophylaxis for heterosexual HIV transmission in Botswana. N Engl J Med. 2012;367(5):423-34

17. Grohskopf LA, Chillag KL, Gvetadze R, et al. Randomized trial of clinical safety of daily oral tenofovir disoproxil fumarate among HIV-uninfected men who have sex with men in the United States. J Acquir Immune Defic Syndr. 2013;64(1):79-86.

18. Baeten JM, Donnell D, Ndase $\mathbf{P}$, et al. Antiretroviral prophylaxis for HIV prevention in heterosexual men and women. N Engl J Med. 2012;367(5):399-410.

19. Choopanya K, Martin M, Suntharasamai P, et al. Antiretroviral prophylaxis for HIV infection in injecting drug users in Bangkok, Thailand (the Bangkok Tenofovir Study): a randomised, double-blind, placebo-controlled phase 3 trial. Lancet. 2013;381(9883):2083-90.

20. Marrazzo JM, Ramjee G, Richardson BA, et al. Tenofovir-based preexposure prophylaxis for HIV infection among African women. N Engl J Med. 2015;372:509-18.

21. Van Damme L, Corneli A, Ahmed K, et al. Preexposure prophylaxis for HIV infection among African women. N Engl J Med. 2012;367:411-22.

22. AVAC: Global Advocacy for HIV Prevention. AVAC report 2013: Research \& reality. Available at: http://www.avac.org/sites/default/files/resourcefiles/AVAC\%20Report\%202013 0.pdf. Accessed September 29, 2016.

23. Anderson PL, Glidden DV, Liu A, et al. Emtricitabine-tenofovir concentrations and pre-exposure prophylaxis efficacy in men who have sex with men. Sci Transl Med. 2012;4(151):151ra125.

24. McCormack S, Dunn DT, Desai M, et al. Pre-exposure prophylaxis to prevent the acquisition of HIV-1 infection (PROUD): effectiveness results from the pilot phase of a pragmatic open-label randomized trial. Lancet 2016;387(10013):53-60.

25. Volk JE, Marcus JL, Phengrasamy T, et al. No new HIV infections with increasing use of HIV preexposure prophylaxis in a clinical practice setting. Clin Infect Dis. 2015;61(10):1601-3.

26. Marcus JL, Hurley LB, Hare CB, et al. HIV preexposure prophylaxis: Adherence and discontinuation in clinical practice. Conference on retroviruses and opportunistic infections [abstract 894]. Boston, Massachusetts: 2016; 22-25 February.

27. Grant RM, Anderson PL, McMahan V, et al. Uptake of pre-exposure prophylaxis, sexual practices, and HIV incidence in men and transgender women who have sex with men: a cohort study. Lancet Infect Dis. 2014; 14(9):820-9.

28. Hosek S, Rudy B, Landovitz R, et al. An HIV pre-exposure prophylaxis (PrEP) demonstration project and safety study for young men who have sex with men in the United States (ATN 110). IAS Conference on HIV Pathogenesis, Treatment \& Prevention [abstract TUAC0204LB]. Vancouver, Canada: 2015; 19-22 July.

29. Patterson KB, Prince HA, Kraft E, et al. Penetration of tenofovir and emtricitabine in mucosal tissues: implications for prevention of HIV-1 transmission. Sci Transl Med. 2011;3(112):112re4.

30. Centers for Disease Control and Prevention. Pre-exposure prophylaxis for the prevention of HIV infection in the United States - 2014: A clinical practice guideline. Available at: http://www.cdc.gov/hiv/pdf/ prepguidelines2014.pdf. Accessed September 29, 2016.
31. Amico KR, Mansoor LE, Corneli A, Torjesen K, Van Der Straten A. Adherence support approaches in biomedical HIV prevention trials: experiences, insights and future directions from four multisite prevention trials. AIDS Behav. 2013;17(6):2143-55.

32. Koenig HC, Mounzer K, Daughtridge GW, et al. Urine assay for tenofovir to monitor adherence to tenofovir-emtricitabine as PrEP. Conference on retroviruses and opportunistic infections [abstract 975]. Seattle, Washington: 2015; 23-26 February.

33. Horberg M, Raymond B. Financial policy issues for HIV pre-exposure prophylaxis. Am J Prev Med. 2013;44(1):S125-8.

34. Gilead Sciences. U.S. patient access. Truvada for PrEP medication assistance program. Available at: http://www.gilead.com/responsibility/us-patient-access/truvada\%20for\%20prep\%20medication\%20assistance\%20program. Accessed September 29, 2016.

35. Grant RM, Liegler T. Weighing the risk of drug resistance with the benefits of HIV preexposure prophylaxis. J Infect Dis. 2015;211(8): 1202-4.

36. Weis JF, Baeten JM, Mccoy CO, et al. Preexposure prophylaxis-selected drug resistance decays rapidly after drug cessation. AIDS. 2016;30(1):31-5

37. Dimitrov D, Boily MC, Brown ER, Hallett TB. Analytic review of modeling studies of ARV based PrEP interventions reveals strong influence of drugresistance assumptions on the population-level effectiveness. PLoS ONE. 2013;8(11):e80927.

38. van de Vijver DA, Nichols BE, Abbas UL, et al. Pre-exposure prophylaxis (PrEP) will have a limited impact on the prevalence of HIV-1 drug resistance in sub-Saharan Africa: a comparison of mathematical models. AIDS. 2013;27(18):2943-51

39. Desai M, Gafos M, Dolling D, McCormack S, Nardone A. Healthcare providers' knowledge of, attitudes to and practice of pre-exposure prophylaxis for HIV infection. HIV Med. 2016;17(2):133-42.

40. Knox DC, Tan DH, Harrigan PR, Anderson PL. HIV-1 infection with multiclass resistance despite preexposure prophylaxis (PrEP). Conference on retroviruses and opportunistic infections [abstract 169aLB]. Boston, Massachusetts: 2016; 22-25 February.

41. Glidden DV, Rivet AK, Liu AY, et al. Symptoms, side effects and adherence in the iPrEx open-label extension. Clin Infect Dis. 2016;62(9):1172-7.

42. Gandhi M, Glidden DV, Liu AY, et al. Higher cumulative TFV/FTC levels in PrEP associated with decline in renal function. Conference on retroviruses and opportunistic infections [abstract 866]. Boston, Massachusetts: 2016; 22-25 February.

43. Mugwanya KK, Wyatt C, Celum C, et al. Changes in glomerular kidney function among HIV-1-uninfected men and women receiving emtricitabinetenofovir disoproxil fumarate preexposure prophylaxis: a randomized clinical trial. JAMA Intern Med. 2015;175(2):246-54.

44. Solomon MM, Lama JR, Glidden DV, et al. Changes in renal function associated with oral emtricitabine/tenofovir disoproxil fumarate use for HIV pre-exposure prophylaxis. AIDS. 2014;28(6):851-9.

45. Mulligan K, Glidden DV, Anderson PL, et al. Effects of emtricitabine/ tenofovir on bone mineral density in HIV-negative persons in a randomized, double-blind, placebo-controlled trial. Clin Infect Dis. 2015;61(4):572-80.

46. Kasonde M, Niska RW, Rose $\mathbf{C}$, et al. Bone mineral density changes among HIV-uninfected young adults in a randomised trial of pre-exposure prophylaxis with tenofovir-emtricitabine or placebo in Botswana. PLoS ONE. 2014;9(3): 1-9.

47. Grant PM, Cotter AG. Tenofovir and bone health. Curr Opin HIV AIDS. 2016;11(3):326-32

48. Liu AY, Vittinghoff E, Sellmeyer DE, et al. Bone mineral density in HIVnegative men participating in a tenofovir pre-exposure prophylaxis randomized clinical trial in San Francisco. PLoS ONE. 2011;6(8):e23688.

49. Grant R, Mulligan, K., McMahan V, et al. Recovery of bone mineral density after stopping oral HIV preexposure prophylaxis. Conference on retroviruses and opportunistic infections [abstract 48LB]. Boston, Massachusetts: 2016;22-25 February.

50. Sax PE, Wohl D, Yin MT, et al. Tenofovir alafenamide versus tenofovir disoproxil fumarate, coformulated with elvitegravir, cobicistat, and emtricitabine, for initial treatment of HIV-1 infection: two randomized, double-blind, phase 3, non-inferiority trials. Lancet. 2015;385(9987):2606-16.

51. Garrett KL, Cottrell ML, Prince HM, et al. Concentrations of TFV and TFVdp in female mucosal tissues after a single dose of TAF. Conference on retroviruses and opportunistic infections [abstract 102LB]. Boston, Massachusetts: 2016; 22-25 February.

52. Spinner CD, Boesecke C, Zink A, et al. HIV pre-exposure prophylaxis (PrEP): a review of current knowledge of oral systemic HIV PrEP in humans. Infection. 2016;44(2): 151-8. 
53. Marcus JL, Glidden DV, Mayer KH, et al. No evidence of sexual risk compensation in the iPrEx trial of daily oral HIV preexposure prophylaxis. PLoS ONE. 2013;8(12):e81997.

54. Liu AY, Cohen SE, Vittinghoff E, et al. Preexposure prophylaxis for HIV infection integrated with municipal- and community-based sexual health services. JAMA Intern Med. 2016;176(1):75-84.

55. Golub SA, Pena S, Boonrai K, Douglas N, Hunt M, Radix A. STI data from community-based PrEP implementation suggest changes to CDC guidelines. Conference on retroviruses and opportunistic infections [abstract 869]. Boston, Massachusetts: 2016; 22-25 February.

56. Flash C, Landovitz R, Giler RM, et al. Two years of Truvada for preexposure prophylaxis utilization in the U.S. J Int AIDS Soc. 2014;17(4 Suppl 3):19730.

57. Delaney KP, Sanchez T, Bowles K, Oraka E, DiNenno E, Sullivan P. Awareness and use of PrEP appear to be increasing among internet samples of US MSM. Conference on retroviruses and opportunistic infections [abstract 889]. Boston, Massachusetts: 2016; 22-25 February.

58. Mayer K, Krakower D, Levine K, et al. Significant increases in HIV preexposure prophylaxis (PrEP) uptake in Boston, a Boston Community Health Center in 2014: who are the recent users? In: 8th IAS Conference on HIV Pathogenesis, Treatment and Prevention. Vancouver; 2015.

59. Smith DK, Van Handel M, Wolitski RJ, et al. Vital signs: estimated percentages and numbers of adults with indications for preexposure prophylaxis to prevent HIV acquisition - United States, 2015. MMWR Morb Mortal Wkly Rep. 2015;64(46):1291-5.

60. Association of American Medical Colleges. Center for Workforce Studies. 2014 Physician Specialty Data Book. Available at: https://members.aamc. org/eweb/upload/Physician\%20Specialty\%20Databook\%202014.pdf. Accessed September 29, 2016.

61. Blumenthal J, Jain S, Krakower D, et al. Knowledge is power! Increased provider knowledge scores regarding pre-exposure prophylaxis (PrEP) are associated with higher rates of PrEP prescription and future intent to prescribe PrEP. AIDS Behav. 2015;19(5):802-10.

62. Mimiaga MJ, White JM, Krakower DS, Biello KB, Mayer KH. Suboptimal awareness and comprehension of published preexposure prophylaxis efficacy results among physicians in Massachusetts. AIDS Care. 2014;26(6):684-93.

63. Edelstein ZR, Salcuni PM, Restar A, et al. Early adopters and incident PrEP prescribing in a detailing campaign, 2014-2015. Conference on retroviruses and opportunistic infections [abstract 892]. Boston, Massachusetts: 2016; 22-25 February.

64. Mayer K, Safren S, Haberer J, et al. Project PrEPare: high levels of medication adherence with continued condomless sex in U.S. men who have sex with men in an oral PrEP adherence trial. HIV Research for Prevention [abstract OA07.06 LB]. Cape Town, South Africa: 2014; 28-31 October.

65. Sherman MD, Kauth MR, Shipherd JC, Street RL Jr. Communication between VA providers and sexual and gender minority veterans: a pilot study. Psychol Serv. 2014;11(2):235-42.
66. Patel R, Chan P, Nunn A, et al. Missed opportunities to prescribe PrEP by primary care physicians in Saint Louis. Conference on retroviruses and opportunistic infections [abstract 891]. Boston, Massachusetts: 2016; 22-25 February.

67. Lanier Y, Castellanos T, Barrow RY, Jordan WC, Caine V, Sutton MY. Brief sexual histories and routine HIV/STD testing by medical providers. AIDS Patient Care STDs. 2014;28(3):113-20.

68. Smith DK, Pals SL, Herbst JH, Shinde S, Carey JW. Development of a clinical screening index predictive of incident HIV infection among men who have sex with men in the United States. J Acquir Immune Defic Syndr. 2012;60(4):421-7.

69. Mayer KH, Krakower DS. Editorial commentary: scaling up antiretroviral preexposure prophylaxis: moving from trials to implementation. Clin Infect Dis. 2015;61(10): 1598-600.

70. U.S. Department of Health and Human Services. Health Resources and Services Administration. Approved uniform data system changes for calendar year 2016. (DHHS publication No. PAL 2016-02). Available at: http://bphc.hrsa.gov/datareporting/pdf/pal201602.pdf. Accessed September 29, 2016.

71. U.S. Department of Health and Human Services. Centers for Medicare and Medicaid Services. Electronic Health Record Incentive Program Stage 3 and modifications to meaningful use in 2015 through 2017. (CMS Publication No. 3310-FC and 3311-FCO. Available at: https:// www.gpo.gov/fdsys/pkg/FR-2015-10-16/pdf/2015-25595.pdf. Accessed September 29, 2016.

72. Marcus JL, Volk JE, Pinder J, et al. Successful implementation of HIV preexposure prophylaxis: lessons learned from three clinical settings. Curr HIV/AIDS Rep. 2016;13(2):116-24.

73. Sikkema KJ, Watt MH, Drabkin AS, Meade CS, Hansen NB, Pence BW. Mental health treatment to reduce HIV transmission risk behavior: a positive prevention model. AIDS Behav. 2010;14(2):252-62.

74. Safren SA, Reisner SL, Herrick A, Mimiaga MJ, Stall R. Mental health and HIV risk in men who have sex with men. J Acquir Immune Defic Syndr. 2010;55(Suppl 2):S74-7.

75. Koblin BA, Husnik MJ, Colfax G, et al. Risk factors for HIV infection among men who have sex with men. AIDS. 2006;20(5):731-9.

76. Hirshfield S, Remien RH, Humberstone M, Walavalkar I, Chiasson MA. Substance use and high-risk sex among men who have sex with men: a national online study in the USA. AIDS Care. 2004;16(8):1036-47.

77. Stall R, Paul J, Greenwood G, et al. Alcohol use, drug use and alcoholrelated problems among men who have sex with men: the Urban Men's Health Study. Addiction. 2001;96(11):1589-601.

78. Parker R, Aggleton P. HIV and AIDS-related stigma and discrimination: a conceptual framework and implications for action. Soc Sci Med. 2003;57(1):13-24.

79. Preston DB, D'Augelli AR, Kassab CD, Starks MT. The relationship of stigma to the sexual risk behavior of rural men who have sex with men. AIDS Educ Prev. 2007;19(3):218-30. 